weight, did not flower and died soon after the emergence of the broomrape flowering stalk.

The doubled yields afforded by control of the parasites will more than offset the added cost of both the transgenic seed and the small amount of herbicide, even in underdeveloped countries. This approach should only be used with crops that do not interbreed with related weeds in the same locality. The use of such transgenics represents a necessary stopgap measure until other means are found, as resistance can rapidly evolve to some of these herbicides (such as the ALS inhibitors), but is expected to evolve more slowly to the others ${ }^{2}$.

Daniel M. Joel

Yeshaiahu Kleifeld

Dalia Losner-Goshen

Geza Herzlinger

Department of Weed Research,

Newe-Ya'ar Research Center,

Haifa 31900, Israel

Jonathan Gressel

Department of Plant Genetics,

Weizmann Institute of Science,

Rehovot 76100 , Israel

\title{
Feather asymmetry in Archaeopteryx
}

SIR - After comparing flight feather asymmetry of Archaeopteryx with the asymmetry of several extant birds with various flight styles, 'flapping', 'gliding' and 'flightless', Speakman and Thomson ${ }^{1}$ claim that Archaeopteryx was not capable of sustained flapping flight. In my view, their analysis is flawed and does not support their conclusion.

First, they report asymmetry values for Archaeopteryx feathers that are far too low, and they make a misleading comparison with extant birds. I have examined highquality photographs in two fold-out plates ${ }^{2}$, showing the left and right wing of the Berlin Archaeopteryx specimen at 2.8 and 4.1 times their natural size. The first three feathers are staggered in length, so the anterior margin is entirely free and clearly visible all along the outer half of feathers 1 and 2 in both wings. Also, feather 3 of the right wing has its anterior margin free in the outer part that extends beyond feather 2. The photographs show the ventral side of the wing, so, because of the way feathers are arranged in a bird wing, the rear margin of these feathers is also free and unobscured. At about $25 \%$ of the feather length from the tip, the feather shaft of the four anteriormost primary feathers $(1-4)$ is located $24-34 \%$ of the feather chord behind the leading edge; thus, the trailingvane is 3.11-1.91 times as wide as the leading-vane. This is much more asymmetrical than $41 \%$ of the chord behind the leading edge, or the ratio 1.46 , reported by Speakman and Thomson for primary feathers 4, 5 and 6 of the Berlin specimen.

My asymmetry values are near the lower limit of, but partly inside, the range for 'flapping' and 'gliding' birds (Fig. 2 in ref. 1). Further, Speakman and Thomson's values for extant 'flapping' and 'gliding' birds are from primary feathers 1 or 2 , which are more asymmetrical than feathers further back (Fig. 1 in ref. 1), but they nevertheless compare them with primaries 4, 5 and 6 in Archaeopteryx. I therefore conclude that the feather asymmetry of Archaeopteryx is within the range of modern birds using flapping flight.

Second, on the wing's downstroke, the anteriormost primary feathers of modern birds often separate so that the outer part of each one acts as an aerofoil on its own ${ }^{3}$. The vane asymmetry then comes into operation and effects a proper orientation of the feather to the incident air stream. On random changes of angles of attack, the aerodynamic centre of pressure of a flat plate, or feather, does move fore and aft in such a way that it maintains dynamic stability in pitch, and hence in angle of attack, provided that the span-wise torsion axis lies within the interval $27-35 \%$ of the chord length behind the leading edge ${ }^{4}$. The feather shaft acts as a local torsion axis in any chord-wise profile. When the feather shaft lies ahead of the $27 \%$ chord point, there is a nose-down pitch moment that also tends to match the angle of attack to the incident air-stream.

The crucial feature of Archaeopteryx feather asymmetry is that the shaft of the anteriormost primary feathers is $24-34 \%$ of the chord behind the leading edge, completely ahead of the critical rear limit $35 \%$, as required for self-stability in pitch. Consequently, vane asymmetry in Archaeopteryx primary feathers is pronounced enough to confer automatic pitch control on separated feather tips, and therefore does not indicate a lack of powered flight.

The asymmetry of the first three primaries is what matters most. Because of their staggered length in Archaeopteryx, they all form part of the wing's leading edge and therefore they are the feathers most likely to split apart in flight, acting as aerofoils - actually, leading-edge slats - on their own, with a need for the automatic pitch control that the vane asymmetry gives.

Third, the function of vane asymmetry is linked to the function of feather curvature $^{3}$. Flight feathers in bird wings are per-

\section{Scientific Correspondence}

Scientific Correspondence is intended to provide a forum in which readers may raise points of a scientific character. Priority will be given to letters of fewer than 500 words and five references. manently bent backwards and the feathers are free to rotate in the nose-up sense in their sockets. Nose-down rotation is prevented by the feathers' attachment in their sockets. It is also restricted by the nearestneighbouring feather lying behind it, and partly overlapping the upper side of the trailing vane, so that the feathers are pressed together on the down-stroke. As a result of feather curvature, and less of vane asymmetry, the flight feathers of modern birds rotate in the nose-up sense on the upstroke when this is aerodynamically non-functional, letting air through the wing. This rotation occurs about an axis through the feather base, and because of the shaft curvature, most of the feather vane is behind this axis to provide the required nose-up torque.

Archaeopteryx flight feathers are as strongly curved as those of modern birds ${ }^{3}$. Its feather curvature alone could therefore provide the nose-up moment required for individual feathers to rotate about the axis through the feather base to let air through the wing on the upstroke. Also, the vane asymmetry in Archaeopteryx is pronounced enough to prevent a counteracting nose-down moment to be set up about the local feather shaft on the upstroke. Feather curvature and vane asymmetry in Archaeopteryx are therefore fully consistent with an active, flapping, flight mode.

\section{R. Åke Norberg}

Department of Zoology,

University of Gothenburg,

Medicinaregatan 18,

S-413 90 Gothenburg, Sweden

Speakman and Thomson RePly - Norberg raises several interesting but erroneous comments.

(1) He has measured vane asymmetry for one Archaeopteryx. His measurements exceed those made by $u^{1}$ on two specimens. Unfortunately, many of his measures were made on regions of the feathers which are overlapped. Since measuring asymmetry depends on defining both feather margins, his measurements depend critically on a subjectively inferred position of the hidden margin. Different observers have inferred asymmetry $\left(a_{\mathrm{p}}\right)$ for the overlapped feathers of Archaeopteryx ranging from complete symmetry ${ }^{5}\left(a_{\mathrm{p}}=\right.$ $1.0)$ to extreme asymmetry ${ }^{6}\left(a_{\mathrm{p}}\right.$ is approximately $4-5$ from Fig. 1 in ref. 6). Our measurements are more accurate as they were made on sections of the feathers which do not overlap. The measures he made which were not overlapped refer to the diminutive first and second primaries; these small feathers would not be important in flight. In our measurements of extant birds we ignored the first primary when it was diminutive (less than half the length of the second primary); thus, comparing the diminutive primaries of Archaeopteryx to our sample of extant birds is invalid. 